View Article Online / Journal Homepage / Table of Contents for this issue

1638 CLARKE AND CHAPMAN : THE MEASUREMENT OF $A$

\title{
CLX.-The Measurement of a Homogeneous Chemical Change in a Gas. (The Thermal Decomposition of Ozone.)*
}

\section{By Herbert Edmund Clarke and David Leonard Chapman.}

JudGING by the published experience of those chemists who have made a special study of chemical changes in gases during recent years, and particularly since Victor Meyer investigated the conditions of the union of hydrogen and oxygen, we should doubtless be compelled to conclude that slow pyrogenic changes in gases have their seat of action principally, if not entirely, on the walls of the enclosing vessels, and that any attempt to measure the velocity of change within a gas itself would be unsuccessful, owing to the impossibility of estimating the precise effect of surface.

A moment's consideration, however, will make it clear that if we could be certain that only a small fraction of the total chemical change were confined to the gas not in the neighbourhood of the walls of the containing vessel, then the effect of surface might be almost entirely eliminated by increasing the size of the vessel until the ratio of its internal surface to its volume became very small. The only difficulty in the way of measuring the velocity of a homogeneous chemical change in the gas would then be the practical one attending the use of a vessel the dimensions of which were large enough for the purpose.

In the present communication, we shall show that, as a matter of fact, the influence of surface on the rate of thermal decomposition

* Compare E. Warburg (Sitzungsber. K. Akad. Wiss. Berlin, 1901, 48, 1126), also E. P. Perman and R. H. Greaves (Proc. Roy. Soc., 1908, A, 80, 353). In neither of the above papers is the special point dealt with in the present communication adequately discussed or investigated, the attention of the authors having been directed towards other objects. 
of ozone at $100^{\circ}$ in a glass vessel becomes negligible when the vessel is increased in size to quite moderate and convenient proportions. Our results for the rate of decomposition of ozone under these conditions are therefore the first recorded examples of measurements of the velocity of a strictly homogeneous change in the gaseous state.

If a gas of given composition undergoes slow chemical change in a vessel of volume $V$ and area $A$, the rate of change may be expressed approximately by

$$
K V+k A,
$$

where $K$ is a constant depending on the composition of the gas, and $k$ is another constant depending both on the composition of the gas and the nature of the surface.

The rate of change per unit volume will be

$$
K+\frac{k A}{V}
$$

and if the action is accompanied by alteration of pressure, we shall have

$$
\frac{\delta p}{\delta \tau} \propto K+\frac{k A}{V}
$$

$p$ being the pressure at a time $\tau$.

In our experiments, two vessels, of which the ratios $\frac{A}{V}$ were known to be very different, were filled with the same sample of ozonised oxygen, and heated side by side in a bath of boiling water. The rate of increase of pressure was in all cases about the same in each vessel proving that the term $\frac{k A}{V}$, depending on the nature of the surface, could be neglected, and that the rate of decomposition could be taken as directly proportional to $K$, a constant the value of which at any given temperature and pressure is determined solely by the composition of the gas.

\section{Experimental.}

Oxygen, prepared by heating potassium permanganate, was first passed in a slow stream through aqueous potassium hydroxide, and then dried with concentrated sulphuric acid. The gas was submitted to the action of the silent discharge in a glass ozoniser, all air having been previously swept out of this part of the apparatus by a current of oxygen. To ensure uniformity of composition, the gas issuing from the ozoniser was collected in a large globe over concentrated sulphuric acid, and allowed to stand some time before being used.

The method adopted for filling the vessels with ozonised oxygen and 
for measuring the changes of pressure in each will be understood by reference to Fig. 1.

The bath $M$ contains water, which is raised to the boiling point before the filling of the apparatus is started, and maintained at the same temperature while the pressure measurements are being made, the level of the water in the bath being kept constant.

Ozonised oxygen is forced, under pressure, from the globe in which it has been collected into the tube $x$. The gas then divides into two streams, and, passing the opened taps $m$ and $n$, reaches the immersed vessels $A$ and $B$ by the capillary tubes $o$ and $p$. The displaced air

FIG. 1.

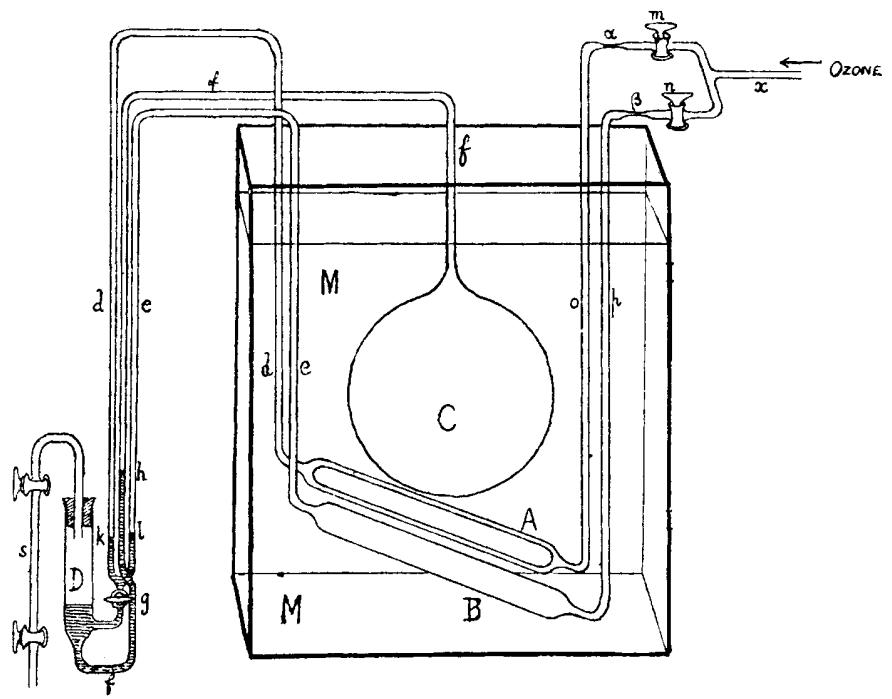

passes along $d$ and $e$, and finally escapes through the sulphuric acid contained in the reservoir $D$, the tap $g$ being open.

When all the air has been swept out of the apparatus, the capillaries $o$ and $p$ are sealed off at the constrictions $a$ and $\beta$, and immediately afterwards the pressure of the air above the sulphuric acid in $D$ is increased by blowing through the tube $s$, so as to drive sulphuric acid into the manometer capillary tubes $d$, $e$, and $f$. A convenient portion of the scale having been chosen, the positions of the sulphuric acid, $k, l, h$, in the tubes are carefully recorded.

The capillary tube $f$ communicates directly with a large globe, $C$, contained in the bath of boiling water, and, since this tube has a volume very small in comparison with that of the globe, any movement of the sulphuric acid in it cannot appreciably alter the pressure of the 
air enclosed in the globe. The perpendicular distance between $h$ and $k$ is therefore a direct measurement, in terms of a column of sulphuric

Fig. 2.

Vessels $I$ and $I I .-\left(A V^{\prime} \mid A^{\prime} V=2 \cdot 24\right)$.

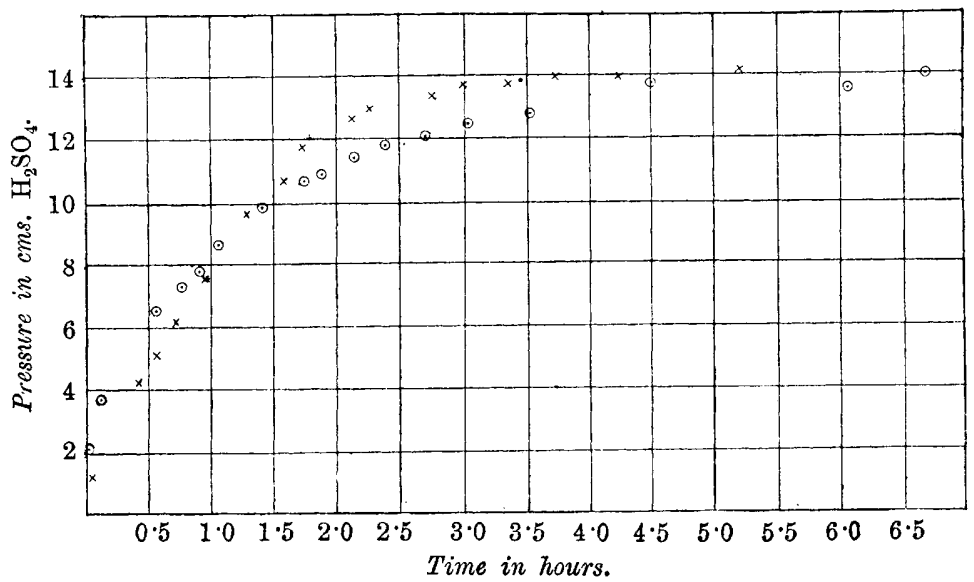

acid, of the difference of pressure in $C$ and $A$. In measuring the variation in this difference of pressure at known intervals during

Fig. 3.

Vessels $I$ and $I I .-\left(A V^{\prime} \mid A^{\prime} V=2 \cdot 24\right)$.

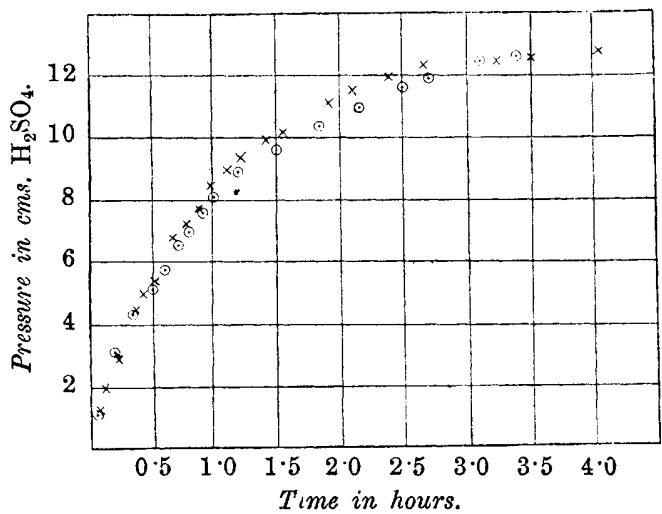

The fact that the curves in Figs. 2 and 3 (the records of the results of two successive experiments with the same vessels) are similar, indicates that the rate of decomposition of the ozone is independent of the length of time that the surface of the glass vessels used has been heated in contact with the de. composing gas.

the progress of the decomposition, the sulphuric acid is always brought to the same point, $k$, so as to keep the volume of gas in $A$ constant. 
1642 CLARKE AND CHAPMAN : THE MEASUREMENT OF A

The readings then furnish a series of numbers from which the rate of increase of pressure in $A$ can be deduced.

Fig. 4.

Vessels II and III.- $\left(A V^{\prime} / A^{\prime} V=2 \cdot 11\right)$.

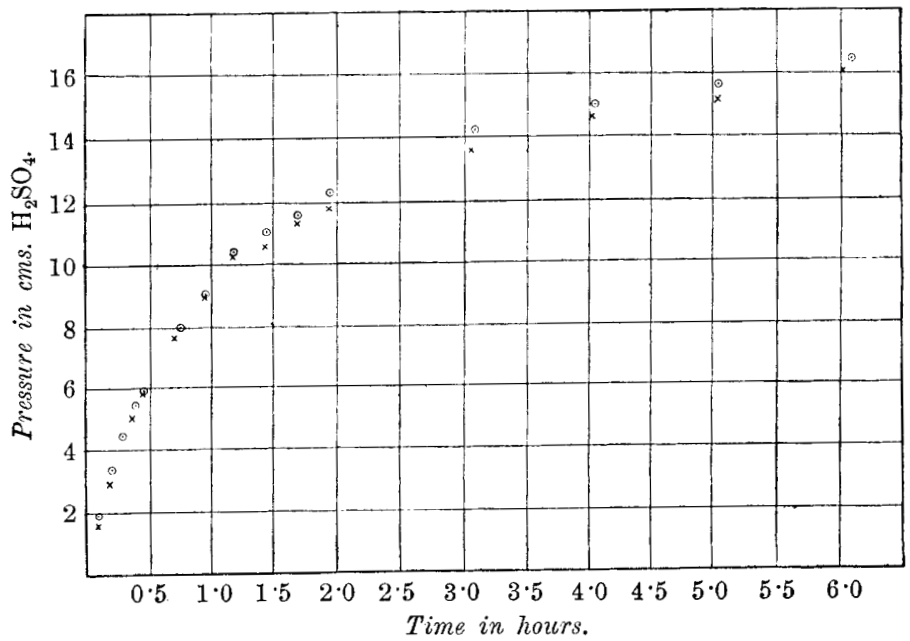

Fig. 5 .

Vesscls III and $I V .-\left(A V^{\prime} / A^{\prime} V=6 \cdot 71\right)$.

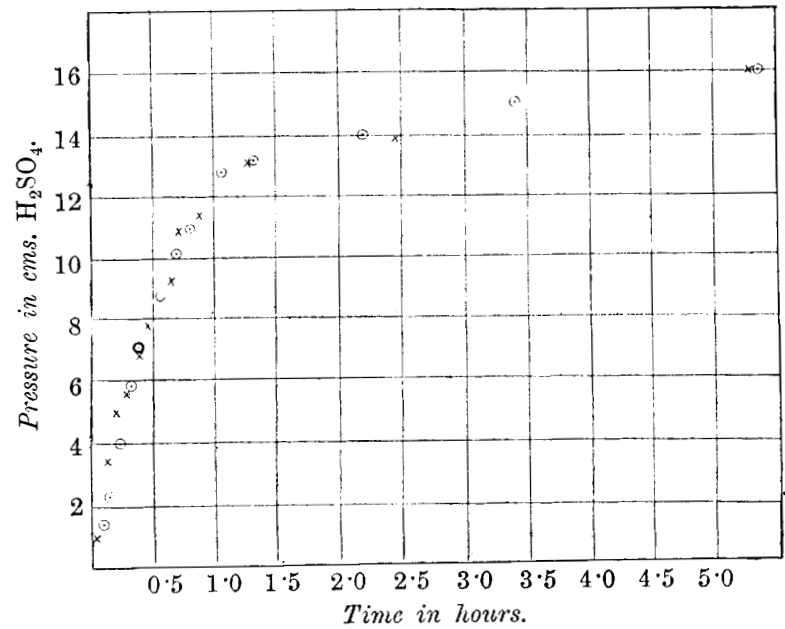

In the same way the increase of pressure in $B$ can be found.

The curves obtained by plotting the readings are shown in Figs. 2 
to 8 . For convenience in reference, the points corresponding to the increments of pressure in the larger vessel are indicated by circles, and those corresponding to the increments in the other by crosses. The

Fig. 6.

Vessels III and IV.- $\left(A V^{\prime} / A^{\prime} V=6 \cdot 71\right)$.

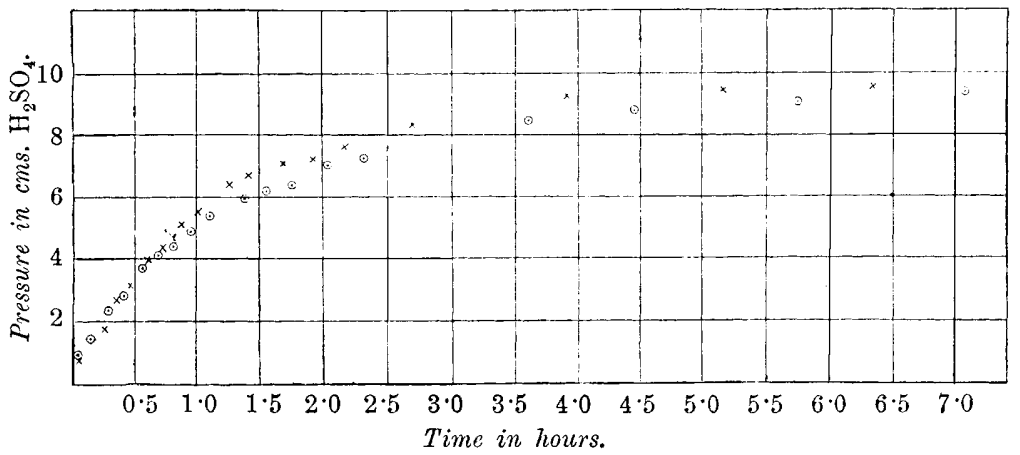

abscissæ are the times in hours counting from the first reading, and the ordinates are the increments of pressure in centimetres of sulphuric acid.

FIG. 7.

$V$ essels $I I$ and $V .-\left(A V^{\prime} / A^{\prime} V=3 \cdot 92\right)$.

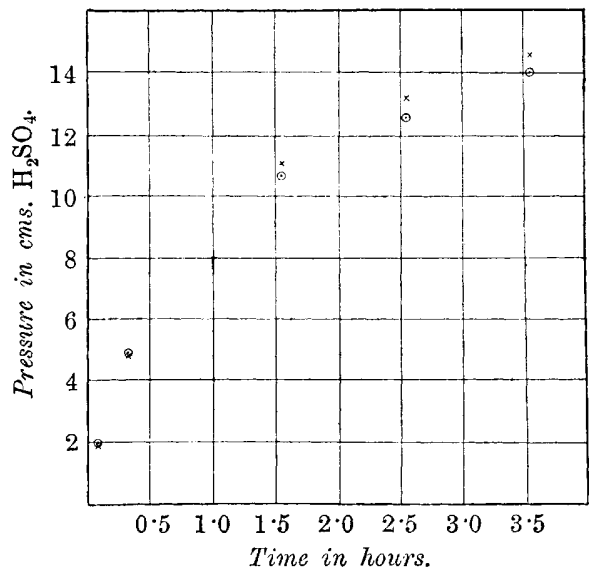

If reference be made to these diagrams, it will be seen that the pressures in both vessels increase at the same rate, although the value of the ratio $\frac{A}{V}$ for one vessel varies in the different experiments 
between twice and seven times that of the same ratio for the other.

We shall not discuss the question of the order of the reaction until the influence on its rate of gaseous impurities, such as nitrogen and aqueous vapour, has been quantitatively investigated.

\section{Dimensions of Vessels Used.}

In the experiments, five different German-glass vessels have been used, the dimensions of which are given in the following tables:

\begin{tabular}{|c|c|c|c|c|}
\hline & & $V$. & A. & $A / V$. \\
\hline No. & Form. & c.c. & sq. cms. & $\mathrm{cms}^{-1}$. \\
\hline I. $\ldots \ldots \ldots \ldots \ldots$ & Cylinder & $138 \cdot 5$ & 216.8 & $1 \cdot 56$ \\
\hline II & ", & $\begin{array}{r}30.0 \\
\end{array}$ & $\begin{array}{l}104 \cdot 6 \\
216 \cdot 2\end{array}$ & $\begin{array}{l}3 \cdot 49 \\
1 \cdot 65\end{array}$ \\
\hline V. .... & Sphere & 161.5 & $\begin{array}{l}216 \cdot 2 \\
143 \cdot 4\end{array}$ & 0.89 \\
\hline
\end{tabular}

Fig. 8.

Vessels $I I$ and $V .-\left(A V^{\prime} \mid A^{\prime} V=3.92\right)$.

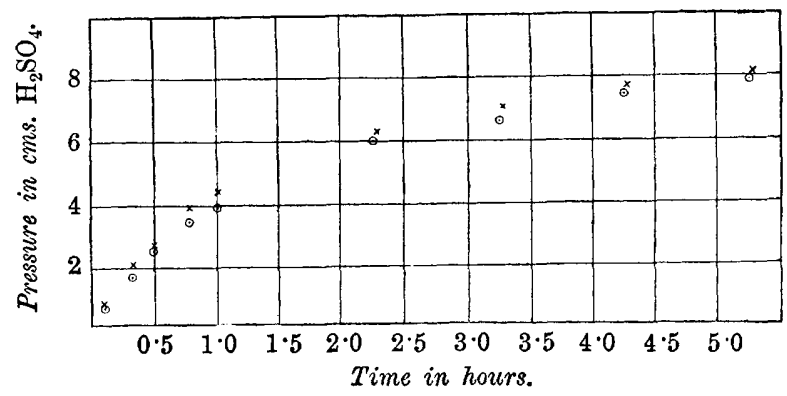

$A$ and $V$ are the internal surface and volume respectively. Vessel No. IV was a cylinder $28 \mathrm{cms}$. long, enclosing a shorter concentric cylinder of less radius, closed at both ends, and $26.2 \mathrm{cms}$. in length. The volume and surface dimensions of this vessel are given below, $V_{1}$ and $A_{1}$ being respectively the internal volume and area of the outer cylinder, and $V_{2}$ and $A_{2}$ the external volume and area of the inner cylinder. The ratio of the total surface in contact with the gas to the volume of the gas is therefore given by $\frac{A_{1}+A_{2}}{V_{1}-V_{2}}$.

No. Form. $\quad V_{1}$. $\quad V_{2} . \quad A_{1} . \quad A_{2} . \quad \frac{A_{1}+A_{2}}{\bar{V}_{1}-V_{2}}$

$\begin{array}{lcccccc}\text { IV. ...... } & \begin{array}{c}\text { Cylinder within } \\ \text { cylinder }\end{array} & \text { c.c. } & \text { c.c. } & \text { sq. cms. } & \text { sq. cms. } & \text { cms }^{-1} \text {. } \\ & 128.5 & 93.5 & 212.4 & 175.5 & 11.08\end{array}$

If in an experiment the ratios of internal surfaces to volumes in the 
THE PREPARATION OF DISULPHIDES. PART IV. 1645

two vessels be $\frac{A}{V}$ and $\frac{A^{1}}{V^{1}}$, the expression $\frac{A V^{1}}{A^{1} V}$ gives the number of times the surface per unit volume of the first vessel is greater than that of the second.

With each curve the corresponding value of the ratio $\frac{A V^{1}}{A^{1} V}$ is given.

The results demonstrate that under suitable conditions the decomposition of ozone is practically a homogeneous change.

We desire to offer our best thanks to Mr. Underhill, of this College, for his kindly assistance in the later stages of the work.

Sir Leoline Jenkins Laboratories,

Jesus College,

OXFORD. 